\title{
Artificial intelligence to predict task activation from resting state fMRI
}

\author{
Burak Kocak $^{1}$ (D)
}

Received: 1 March 2021 / Accepted: 1 April 2021 / Published online: 7 May 2021

(C) European Society of Radiology 2021

Functional magnetic resonance imaging (fMRI) is a noninvasive tool to monitor dynamic changes in the neural metabolism of the brain through detecting changes in oxygenation. Since the early 1990s, fMRI has become an essential tool for the evaluation of neuronal activity of the brain. Not only cognitive tasks but also resting state causes alterations of neuronal activity. There are two main types of fMRI: task-based and resting state. In routine clinical practice, fMRI is mostly performed with a task-based approach. This is mostly due to less complex analysis is involved after preprocessing of taskbased MRI and easiness of interpretation. In this method, patients are requested to perform certain cognitive or sensorimotor tasks, such as finger tapping, tactile stimulation, lip-puckering, tongue movement, foot movement, auditory responsive naming, and so on. It has been widely used for presurgical mapping of the important brain regions to evaluate the potential risk and avoid sensorimotor deficits. Task-based fMRI might be unsatisfactory in patients who cannot perform such active tasks, pediatric population, uncooperative patients, etc. In such circumstances, other methods are available, for instance, passive tasks [1]. Furthermore, resting state fMRI is an alternative for this purpose [2]. In contrast to task-based fMRI imaging, resting state fMRI is performed without a stimulus, that is, at complete rest. However, even with promising results and easy settings, its clinical acceptance for preoperative mapping has been hindered by important methodological problems such as the complexity of the post-processing, standardization issues, and difficulties in interpretation [3]. Following pre-preprocessing steps, task-based fMRI is usually analyzed with $t$-test, correlation analysis, generalized

This comment refers to the article available at https://oi.org/10.1007/ s00330-021-07825-w.

Burak Kocak

drburakkocak@gmail.com

1 Department of Radiology, Basaksehir Cam and Sakura City Hospital, Basaksehir, 34480 Istanbul, Turkey linear modeling, or multivariate pattern analysis [4]. On the other hand, resting state fMRI is usually analyzed with independent component analysis, which is a model-free approach, in other words, having no prior assumptions [4]. However, independent component analysis has some inherent drawbacks [4]. First, it is a very complicated representation of fMRI data so that interpreting group differences becomes difficult. Second, dimension of the components is specified by users such that it might be biased. Third, designation of the components into noise or actual signal might be affected by the interpretation bias. Other traditional methods are seedbased analysis, clustering, generalized linear modeling, and so on [4]. As a subfield of artificial intelligence, machine learning is the study of algorithms to make prediction models without explicit programming in advance. Machine learning algorithms iteratively learn from the training data to improve the predictive performance over time. Several machine learning algorithms exist and many more are under development. Of them, inspired by biological neurons, artificial neural networks are a special type of machine learning and the main building block of the most advanced machine learning algorithms like deep learning. In some previous fMRI works, it has been reported that machine learning algorithms can predict better than traditional ones $[5,6]$.

Published in this issue, a study by Niu et al [7] aimed to evaluate the predictive performance of machine learning, specifically an artificial neural network-based method, in presurgical mapping of hand motor areas using resting state fMRI data of the patients with perirolandic brain tumor. The authors included 109 adult patients with a brain tumor around perirolandic cortex (meningioma, glioma, and brain metastases) and 47 adult healthy controls. In their study, the neural network model trained on healthy controls was applied to predict hand motor activation in patients with brain tumor. Neural network-based maps performed better than the conventional independent component analysis and generalized linear modeling approaches in their single center and internally validated study. Furthermore, the neural network model 
detected the hand motor activation very well, corresponding to both active and passive tasks on task-based fMRI. Many previous works used linear approaches to predict activations from the resting state fMRI. However, the underlying assumptions of the linear models are not always appropriate for fMRI data. In this respect, the use of non-linear complex algorithms seems to be promising. Furthermore, they reported no significant differences between passive and active tasks in their activation patterns. This finding was important because head motion related to these tasks is considered a challenge in fMRI acquisition, causing misleading conclusions. They suggest that the machine learning-based prediction would lessen the impacts of such head motion problems, which is important for patients who cannot perform tasks. Moreover, in line with the previous research, the authors reported that the dice coefficient was significantly lower in patients with glioma, compared to meningioma and metastases. This was probably due to neurovascular uncoupling phenomenon. Therefore, tumor type must be considered in the interpretation of the neural network model for accurate presurgical planning. In summary, the neural network-based machine learning approach to predict hand motor activation based on resting state fMRI data of patients with perirolandic brain tumor is a good contribution to the current literature. Considering the limitations of the study, future research on the subject should focus on gathering higher number of patient data, multi-center collaborations for independent validation, improving dice coefficient, studying other cognitive and sensorimotor tasks, the inclusion of pediatric populations, and comparison with other traditional approaches.

Funding The authors state that this work has not received any funding.

\section{Declarations}

Guarantor The scientific guarantor of this publication is Burak Kocak, MD.
Conflict of interest The authors of this manuscript declare no relationships with any companies whose products or services may be related to the subject matter of the article.

Statistics and biometry Not applicable.

Informed consent Not applicable.

Ethical approval Not applicable.

Methodology

- Editorial commentary

\section{References}

1. Kocak M, Ulmer JL, Sahin Ugurel M et al (2009) Motor homunculus: passive mapping in healthy volunteers by using functional MR imaging-initial results. Radiology 251:485-492. https://doi.org/10. 1148/radiol.2512080231

2. Parker Jones O, Voets NL, Adcock JE et al (2016) Resting connectivity predicts task activation in pre-surgical populations. NeuroImage Clin 13:378-385. https://doi.org/10.1016/j.nicl.2016. 12.028

3. Lang S, Duncan N, Northoff G (2014) Resting-state functional magnetic resonance imaging: review of neurosurgical applications. Neurosurgery 74:453-464; discussion 464-465. https://doi.org/10. 1227/NEU.0000000000000307

4. Chen JE, Glover GH (2015) Functional magnetic resonance imaging methods. Neuropsychol Rev 25:289-313. https://doi.org/10.1007/ s11065-015-9294-9

5. Rasero J, Aerts H, Ortega MO et al (2018) Predicting functional networks from region connectivity profiles in task-based versus resting-state fMRI data. PLoS One 13:e0207385. https://doi.org/10. 1371/journal.pone.0207385

6. Cohen AD, Chen Z, Parker Jones O et al (2019) Regression-based machine-learning approaches to predict task activation using restingstate fMRI. Hum Brain Mapp 41:815-826. https://doi.org/10.1002/ hbm. 24841

7. Niu C, Wang Y, Cohen AD et al (2021) Machine learning may predict individual hand motor activation from resting-state fMRI in patients with brain tumors in perirolandic cortex. Eur Radiol. https:// doi.org/10.1007/s00330-021-07825-w

Publisher's note Springer Nature remains neutral with regard to jurisdictional claims in published maps and institutional affiliations. 